\title{
Structural and Theoretical Investigation of N'-[(E)- (4-Bromophenyl)(Phenyl)Methylidene]-4-Methyl benzene sulfonohydrazide Crystal Prepared by Slow Evaporation Method
}

\author{
V. MOHAN ${ }^{1,2}$, P. MAADESWARAN ${ }^{3, *}$, \\ B. BABU ${ }^{4}$ and J. CHANDRASEKARAN ${ }^{4}$ \\ ${ }^{1}$ Research and Development Center, Bharathiar University, Coimbatore - 641 046, Tamil Nadu, India. \\ ${ }^{2}$ Department of Physics, K.S.Rangasamy College of Technology, \\ Tiruchengode-637215, Tamil Nadu, India. \\ ${ }^{3}$ Department of Energy Studies, Periyar University, Salem-636011, Tamil Nadu, India. \\ ${ }^{4}$ Department of Physics, Sri Ramakrishna Mission Vidyalaya College of Arts and Science, \\ Coimbatore - 641 020, Tamil Nadu, India. \\ *Corresponding author E-mail: maadesphysics@gmail.com \\ http://dx.doi.org/10.13005/ojc/330302
}

(Received: April 19, 2017; Accepted: May 26, 2017)

\section{ABSTRACT}

The N'-[(E)-(4-Bromophenyl) (phenyl)methylidene]-4-methylbenzenesulfonohydrazide molecules were synthesized by condensation method. The synthesized N'-[(E)-(4-Bromophenyl) (phenyl)methylidene]-4- methyl benzene sulfonohydrazide crystal geometric parameters were characterized by single crystal X-ray diffraction analysis. The crystal structure and optimized geometry parameter of N'-[(E)-(4-Bromophenyl)(phenyl)methylidene]-4-methyl benzene sulfonohydrazide molecules were obtained by the B3LYP STO-3gG level of basis set. The Mulliken charges, Highest Occupied Molecular Orbits (HOMO) and Lowest Unoccupied Molecular Orbits (LUMO) analyses have been done in order to calculate the energy gap, Ionization potential, Electron affinity; Global hardness, Chemical potential, global electrophilicity and Molecular electrostatic potential properties for N'-[(E)-(4-Bromophenyl) (phenyl)methylidene]-4-methylbenzenesulfonohydrazid molecules were found out. The calculated HOMO and LUMO energies show that the charge transfer occurs in the N'-[(E)- (4-Bromophenyl)(phenyl)methylidene]-4- methyl benzene sulfonohydrazid molecules for B3LYP STO-3gG basis set.

Keywords: Crystal structure, X-ray diffraction, HOMO LUMO 


\section{INTRODUCTION}

Benzophenone can be used as a photo initiator in UV-curing applications ${ }^{1}$ such as inks, imaging, and clear coatings in the printing industry. Benzophenone prevents ultraviolet (UV) light from damaging scents and colors in products such as perfumes and soaps. This can also be added to plastic packaging as a UV blocker to prevent photo-degradation of the packaging polymers or its contents. Its use allows manufacturers to package the product in clear glass or plastic (such as a PETE water bottle). Without it, opaque or dark packaging would be required. Its derivatives have been investigated extensively for their biological activities such as anti-fungal and anti-inammatory ${ }^{2-7}$.

In present study, Molecular geometry, Optimized parameters, Atomic charges, Mulliken charges, HOMO (highest occupied molecular orbital) and LUMO (Lowest unoccupied molecular orbital) energies, Frontier orbital energy gap, Molecular electrostatic potential, properties are experimental and computed the performance of the computational methods for ab initio B3LYP STO-3gG basis set are compared.

\section{EXPERIMENTAL}

\section{Synthesis}

The 4-Bromoobenzophenone (1 mmol) and tosylhydrazide ( $1 \mathrm{mmol})$ were dissolved in ethanol (50 $\mathrm{ml})$. The reaction mixture was heated under reflux for $3 \mathrm{hr}$ and cooled gradually to room temperature ${ }^{8}$. The reaction mechanism is shown in Fig. 1. Crystals suitable for X-ray diffraction analysis were obtained by slow room temperature evaporation of the solution containing the compound. The as grown crystals of $\mathrm{N}-[(\mathrm{E})-(4-B r o m o p h e n y l)$ (phenyl)methylidene]-4methyl benzene sulfonohydrazide is depicted in Fig. 2.

\section{Computational details}

The quantum chemical calculation of $\mathrm{N}-[(\mathrm{E})$ (4-Bromophenyl) (phenyl)methylidene]-4-methyl benzene sulfonohydrazide has been performed using the B3LYP STO-3gG level of basis set, using the Gaussian 09 Program. The optimized geometries corresponding to the minimum on the potential energy surface have been obtained by solving self- consistent field equation iteratively. The B3LYP STO$3 \mathrm{gG}$ level of basis set was used for HOMO-LUMO analysis, Electrostatic potential (MESP) properties were calculated by Gaussian 09 Program .

\section{Characterization techniques}

Characterization: Single crystal X-ray intensity data of sucrose was collected at room temperature $(T=296 \mathrm{~K})$ on a Bruker X8 KAPPA APEX-II CCD diffractometer equipped with graphite mono chromated Mo Ka radiation. Initial unit cell parameters were obtained from SMART V5.05 software for CCD detector system; Bruker Analytical X-ray Systems, Madison, WI, 1998. Data integration, correction for Lorentz polarization effects and final cell refinement were performed by SAINTPLUS, V5.00 Software for the CCD detector system; Bruker Analytical X-Ray System, Inc.: Madison, WI, 1998. An empirical absorption correction based on the multiple measurements of equivalent reflections was applied using SADABS, Program for absorption correction using SMART CCD based on the method of Blessing. Structure was obtained by a combination of the direct methods and difference Fourier syntheses and refined by full-matrix least-squares on F2 using the SHELXTL.

\section{RESULTS AND DISCUSSION}

\section{Single crystal X-ray diffraction}

$\mathrm{N}$-[(E)-(4-Bromophenyl) (phenyl) methylidene]-4-methylbenzenesulfonohydrazide optimized geometric crystal structure is shown in the Fig. 3 , belongs to the noncentro symmetric monoclinic space group $P 2_{1} / c$ and the cell dimensions are $a=8.4480 \AA, b=19.7198 \AA, c=12.9679 \AA ; \alpha=\gamma=90^{\circ}$ and $\beta=120.046$; and $V=1870.06 \AA^{3}$. The packing diagram in $\mathrm{N}-[(\mathrm{E})-(4-B r o m o p h e n y l)$ (phenyl)methylidene]4-methyl benzene sulfonohydrazide molecule and crystal structure view along the (a) $a$-axis (b) $b$-axis, and (c) $c$-axis is presented in Fig. $4(a-c)$ and $5(a-c)$. The powder XRD pattern is shown in the Fig. 6.

In order to find the most optimized geometric parameters (bond length, bond angle and dihedral angles), the energy calculation are carried out for $\mathrm{N}^{\prime}$-[(E)-(4-Bromophenyl) (phenyl)methylidene]-4methylbenzenesulfonohydrazide, using B3LYP STO$3 g G$ basis set value is given in the Table (1-3). The hydrazones molecules are attracted considerable 
attention due to their biological activities and crystal structures of these compounds were reported ${ }^{2-7}$. Benzophenone and its derivatives have also been extensively investigated for their biological activities such as anti-fungal and anti-inflammatory. In present work, the title compound, $\mathrm{C}_{20} \mathrm{H}_{17} \mathrm{BrN}_{2} \mathrm{O}_{2} \mathrm{~S}$, was<smiles>Cc1ccc(S(=O)(=O)NNC(c2ccccc2)c2ccc(Br)cc2)cc1</smiles>

$N$-[(4-bromophenyl)(phenyl)methyl]-4-methylbenzenesulfonohydrazide

Fig. 1: N'-[(E)-(4-Bromophenyl)(phenyl)methylidene]-4-methylbenzenesulfonohydrazide molecules synthesis reaction

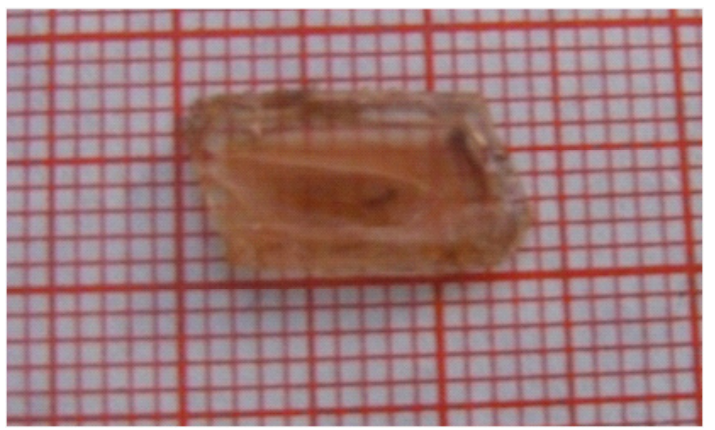

Fig. 2: As grown crystals of N'-[(E)-(4Bromophenyl)(phenyl)methylidene]-4methylbenzenesulfonohydrazide

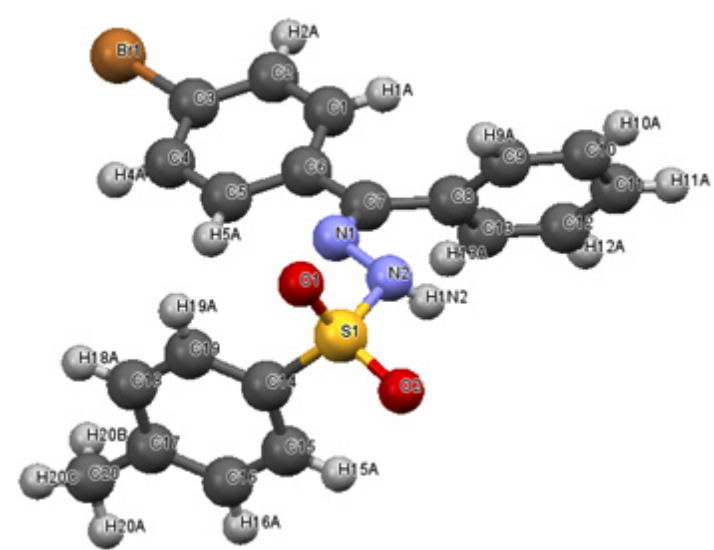

Fig. 3: Geometric structures of N'-[(E)(4-Bromophenyl)(phenyl)methylidene]-4methylbenzenesulfonohydrazide synthesized by Schiff base condensation reaction between 4-bromobenzophenone and tosyl hydrazine were used synthesized by N'-[(E)-(4-Bromophenyl) (phenyl)methylidene]-4-methyl benzene sulfono hydrazide molecules. The optimized geometric structure of molecule (Fig. 3) the bromo substituted with benzene ring (Br1-C3) forms bond length 1.8986 ̊̊and $1.8973 \AA$ for experimental and B3LYP STO-3gG basis set values respectively. The bromo benzophenone linked bridge tosyl hydrazine group (N1-N2) bond length is $1.4014 \AA$ and $1.4519 \AA$ experimental and theoretical values. The bond length of benzene ring carbon and hydrogen bond of (C2-H2A), (C10-H10A), (C12-H12A), $(\mathrm{C} 13-\mathrm{H} 13 \mathrm{~A})$ and $(\mathrm{C} 20-\mathrm{H} 20 \mathrm{C})$ values at $0.9499 \AA$, $0.9493 \AA, 0.9509 \AA, 0.9506 \AA, 0.9497 \AA$ \& $0.9800 \AA$ and $1.0977 \AA$, $1.0984 \AA$, $1.0984 \AA$, $1.0985 \AA$, $1.0985 \AA$ \& $1.1008 \AA$ for experimental and theoretical values. Experimental values as compare to those theoretical values, more or less equal values are get. The N'-[(E)-(4- Bromophenyl)(phenyl) methylidene]-4-methyl benzene sulfonohydrazide molecules hydro-carbon of benzene ring are $(\mathrm{H} 1 \mathrm{~A}$ C1-C2), (H1A-C1-C6), (C8-C9-H9A), (H9A-C9-C10), (C8-C13-H13A), (C12-C13-H13A) (H16A-C16-C17) (H18A-C18-C19) (C14-C19-H19A) and (C18-C19$\mathrm{H} 19 \mathrm{~A})$ bond angle values at $119.50^{\circ}, 119.52^{\circ}$, $119.94^{\circ}, 119.87^{\circ}, 119.82^{\circ}, 119.86^{\circ}, 119.24^{\circ}$, $119.73^{\circ}, 120.16^{\circ} \& 120.10^{\circ}$ and $119.3254^{\circ}$, $119.6576^{\circ}, 119.3914^{\circ}, 119.9437^{\circ}, 119.3903^{\circ}$, $119.9368^{\circ}, 119.6115^{\circ}, 119.5452^{\circ}, 120.7595^{\circ} \&$ 
$120.8558^{\circ}$ for experimental and B3LYP STO-3gG basis set values respectively. The bromine substituted benzene ring (Br1-C3-C4) $119.81^{\circ}$ and $120.2469^{\circ}$ for both values. The bond angle of crystal, molecules are linked through nitrogen and carbon (N1-C7-C6) values at $115.65^{\circ}$ and $115.4307^{\circ}$ for experimental and theoretical values. The molecules, bond angle between two benzene rings (C5-C6-C7) (C6-C7-C8) (C7-C8-C13) and (C11-C12-C13) values at $120.38^{\circ}, 119.60^{\circ}, 120.67^{\circ} \& 119.80^{\circ}$ and $119.8369^{\circ}$, $119.3776^{\circ}, 120.3213^{\circ} \& 120.1798^{\circ}$ for experimental
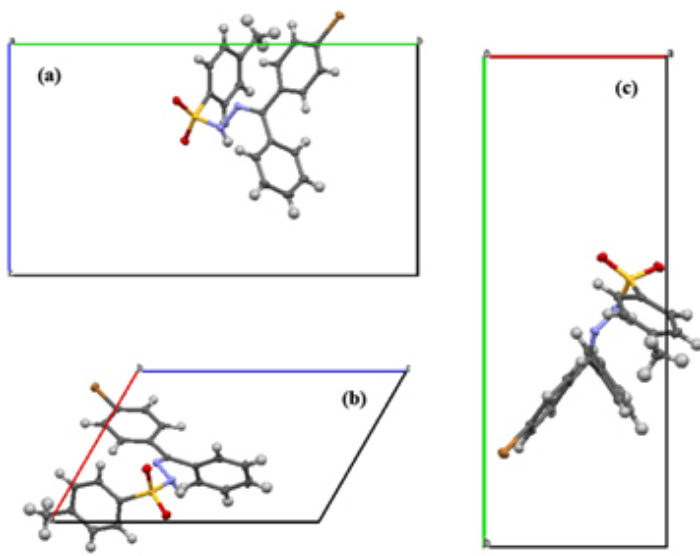

Fig. 4: The molecule packing diagram in N'[(E)-(4-Bromophenyl)(phenyl)methylidene]-4methylbenzenesulfonohydrazide crystal and structure view along the (a) a-axis (b) b-axis (c) c-axis
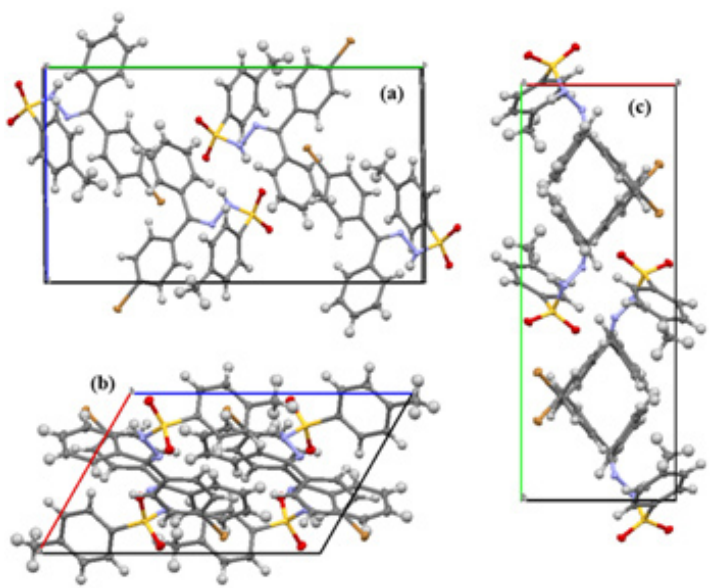

Fig. 5: The crystal packing diagram in N'[(E)-(4-Bromophenyl)(phenyl)methylidene]-4methylbenzenesulfonohydrazide crystal and structure view along the (a) a-axis
Table 1: Optimized geometrical parameters for N'-[(E)-(4-Bromophenyl)(phenyl)methylidene]4-methylbenzenesulfonohydrazide molecules bond length( $(\AA)$

\begin{tabular}{|c|c|c|c|}
\hline $\begin{array}{l}\text { S. } \\
\text { No }\end{array}$ & Bond length & Experimental & $\begin{array}{l}\text { B3LYP } \\
\text { STO-3gG }\end{array}$ \\
\hline
\end{tabular}
1

2
3
4

6
7
8

8

9

10

12

13

14

15

16

17

18

19

20

21

22

23

24

25

26

27

28

29

30

31

32

33

34

35

36

37

38

39

40

41

42

43

44

45

$\begin{array}{ll}1.8986 & 1.8973 \\ 1.4335 & 1.627 \\ 1.4390 & 1.6281\end{array}$

S1-O1

1.4390

1.6281

S1-O2

1.6575

S1-C14 $\quad 1.7589$

2.0742

1.9287

N1-N2 $\quad 1.4014 \quad 1.4519$

N1-C7 $\quad 1.2929 \quad 1.3502$

N2-H1N2 $0.8127 \quad 1.087$

$\begin{array}{lll}\mathrm{C} 1-\mathrm{H} 1 \mathrm{~A} & 0.9499 & 1.0977\end{array}$

$\begin{array}{lll}\mathrm{C} 1-\mathrm{C} 2 & 1.3908 & 1.4057\end{array}$

$\begin{array}{lll}\mathrm{C} 1-\mathrm{C} 6 & 1.3984 & 1.4204\end{array}$

$\begin{array}{lll}\mathrm{C} 2-\mathrm{H} 2 \mathrm{~A} & 0.9493 & 1.0984\end{array}$

$\begin{array}{lll}\mathrm{C} 2-\mathrm{C} 3 & 1.3869 & 1.4163\end{array}$

$\begin{array}{lll}\mathrm{C} 3-\mathrm{C} 4 & 1.3877 & 1.4188\end{array}$

C4-H4A $\quad 0.9500 \quad 1.0983$

$\begin{array}{lll}\mathrm{C} 4-\mathrm{C} 5 & 1.3877 & 1.4024\end{array}$

$\begin{array}{lll}\mathrm{C} 5-\mathrm{H} 5 \mathrm{~A} & 0.9505 & 1.0985\end{array}$

$\begin{array}{lll}\mathrm{C} 5-\mathrm{C} 6 & 1.4009 & 1.4228\end{array}$

$\begin{array}{lll}\mathrm{C} 6-\mathrm{C} 7 & 1.4844 & 1.5079\end{array}$

C7-C8 $\quad 1.4899 \quad 1.5225$

C8-C9 $\quad 1.3974 \quad 1.4198$

$\begin{array}{lll}\mathrm{C} 8-\mathrm{C} 13 & 1.3909 & 1.4205\end{array}$

$\begin{array}{lll}\mathrm{C} 9-\mathrm{H} 9 \mathrm{~A} & 0.9498 & 1.0988\end{array}$

$\begin{array}{lll}\mathrm{C} 9-\mathrm{C} 10 & 1.3925 & 1.4083\end{array}$

C10-H10A $0.9509 \quad 1.0984$

$\begin{array}{lll}\mathrm{C} 10-\mathrm{C} 11 & 1.3843 & 1.4087\end{array}$

$\begin{array}{lll}\mathrm{C} 11-\mathrm{H} 11 \mathrm{~A} & 0.9502 & 1.0984\end{array}$

$\begin{array}{lll}\mathrm{C} 11-\mathrm{C} 12 & 1.3864 & 1.4103\end{array}$

C12-H12A $0.9506 \quad 1.0985$

$\begin{array}{lll}\mathrm{C} 12-\mathrm{C} 13 & 1.3933 & 1.4066\end{array}$

C13-H13A $0.9497 \quad 1.0985$

$\begin{array}{lll}\mathrm{C} 14-\mathrm{C} 15 & 1.3900 & 1.4021\end{array}$

$\begin{array}{lll}\mathrm{C} 14-\mathrm{C} 19 & 1.3887 & 1.4037\end{array}$

C15-H15A $0.9502 \quad 1.1019$

$\begin{array}{lll}\mathrm{C} 15-\mathrm{C} 16 & 1.3970 & 1.4088\end{array}$

$\begin{array}{lll}\mathrm{C} 16-\mathrm{H} 16 \mathrm{~A} & 0.9503 & 1.0989\end{array}$

C16-C17 $1.3963 \quad 1.417$

C17-C18 $1.3965 \quad 1.4175$

$\begin{array}{lll}\mathrm{C} 17-\mathrm{C} 20 & 1.5057 & 1.5387\end{array}$

$\begin{array}{lll}\mathrm{C} 18-\mathrm{H} 18 \mathrm{~A} & 0.9502 & 1.0989\end{array}$

$\begin{array}{lll}\mathrm{C} 18-\mathrm{C} 19 & 1.3882 & 1.4097\end{array}$

$\begin{array}{lll}\mathrm{C} 19-\mathrm{H} 19 \mathrm{~A} & 0.9498 & 1.0999\end{array}$

C20-H20A $0.9806 \quad 1.1005$

C20-H20B $0.9801 \quad 1.1042$

$\begin{array}{lll}\mathrm{C} 20-\mathrm{H} 20 \mathrm{C} & 0.9800 & 1.1008\end{array}$

(b) b-axis (c) c-axis 
and B3LYP STO-3gG basis set values respectively. The dihedral angle between three benzene ring of N'[(E)-(4- Bromophenyl)(phenyl)methylidene]-4-methyl benzene sulfonohydrazide molecules in (C8-C9-C10H10A), (C9-C10- C11-H11A), (H12A-C12-C13-C8), (C15-C14-C19-H19A), (H16A-C16-C17-C18), (C16C17-C18-H18A) and (H18A-C18-C19-C14) values at $178.71^{\circ},-178.91^{\circ}, 178.53^{\circ}, 177.79^{\circ}, 176.92^{\circ}$, $-177.25^{\circ} \& 179.84^{\circ}$ and $179.6285^{\circ},-179.7863^{\circ}$, $179.644^{\circ}, 179.2793^{\circ}, 179.5913^{\circ},-179.6164^{\circ} \&$ $179.9329^{\circ}$ for experimental and B3LYP STO-3gG basis set values respectively.

\section{Mulliken population analysis}

The atomic charges in molecules are fundamental to chemistry. For instance, atomic

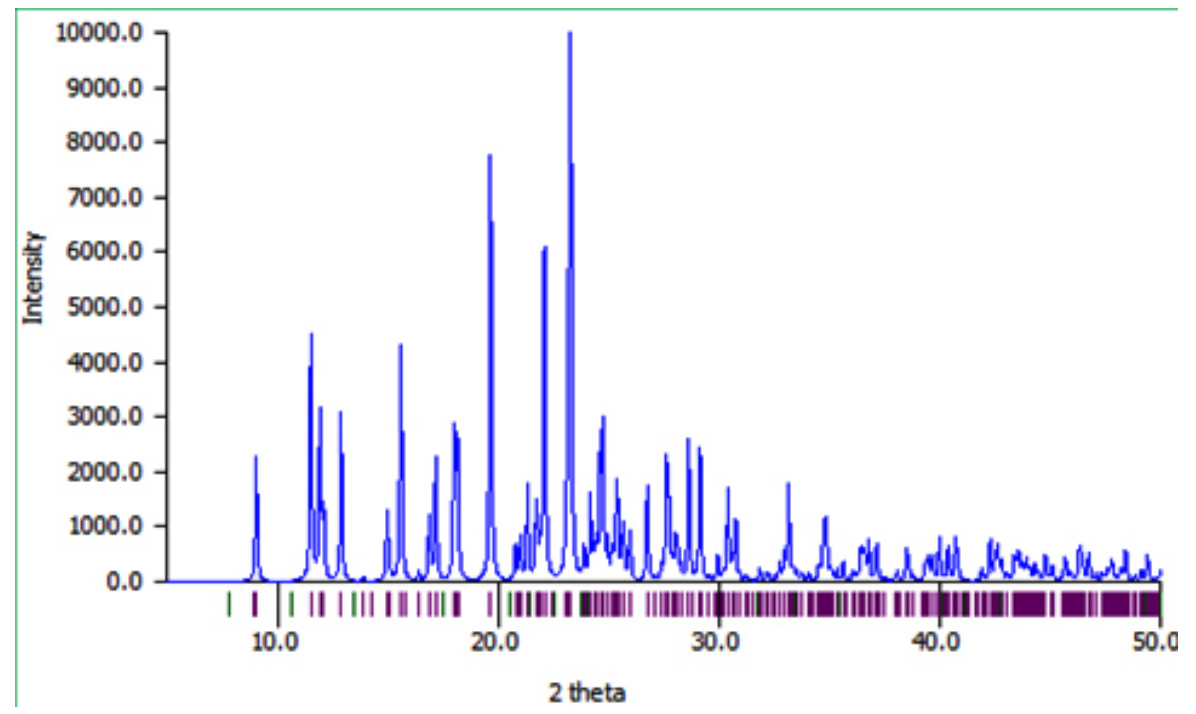

Fig. 6: X-ray diffraction pattern of N'-[(E)-(4-Bromophenyl)(phenyl)methylidene]-4methylbenzenesulfonohydrazide crystal

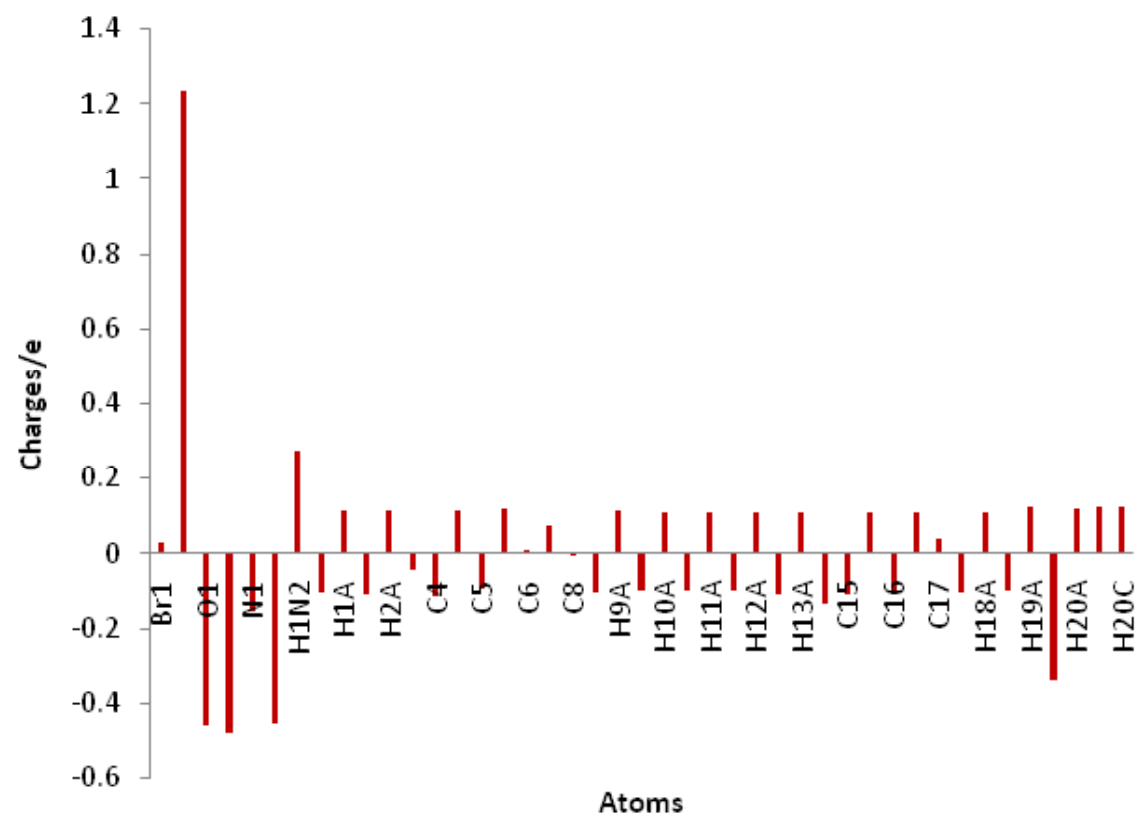

Fig. 7: The charge distribution calculated by the Mulliken method for N'-[(E)-(4-Bromophenyl) (phenyl)methylidene]-4-methylbenzenesulfonohydrazide molecules 

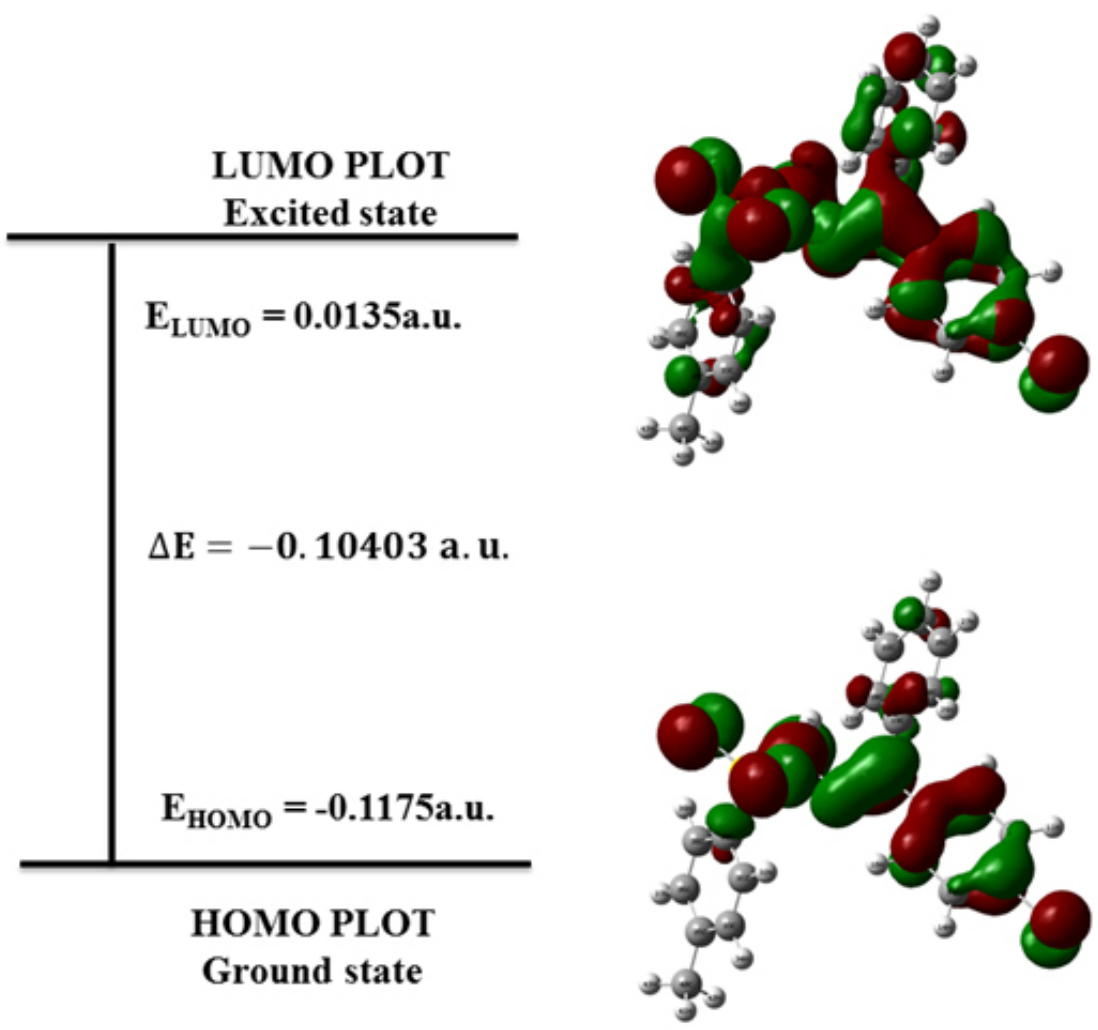

Fig. 8:The atomic orbital compositions of the frontier molecular orbital of N'-[(E)-(4-Bromophenyl) (phenyl)methylidene]-4-methylbenzenesulfonohydrazide molecules

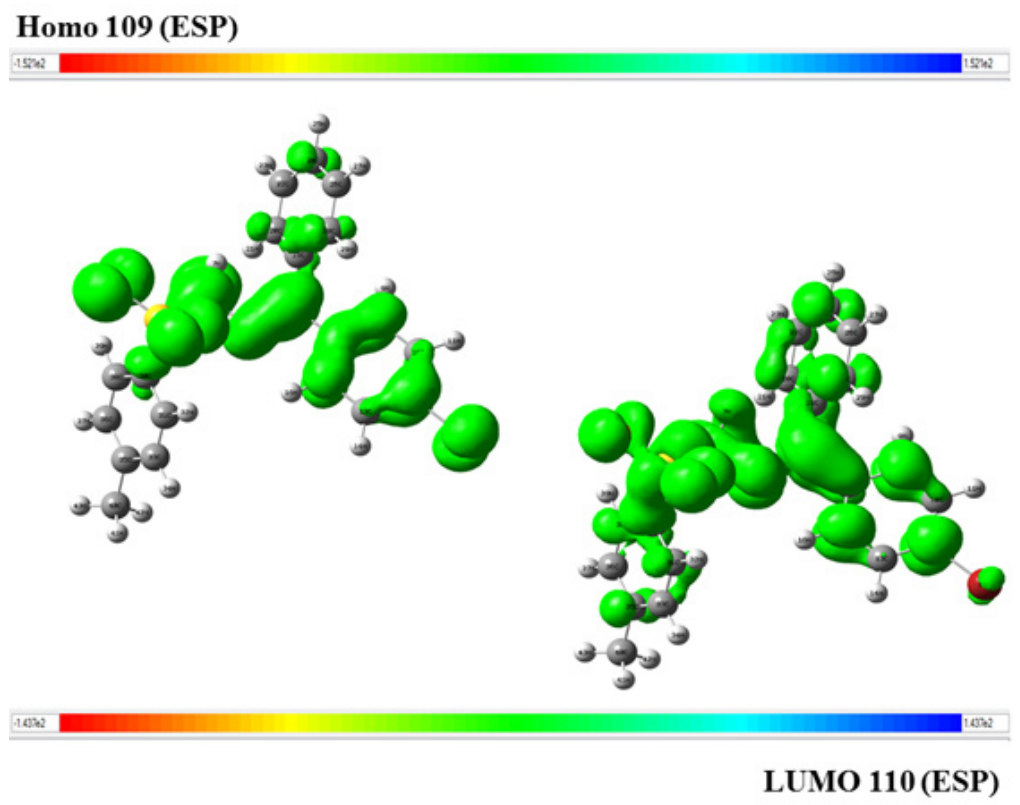

Fig. 9: The Electrostatic potential of diagram in N'-[(E)-(4-Bromophenyl) (phenyl) methylidene] -4-methylbenzenesulfonohydrazide 


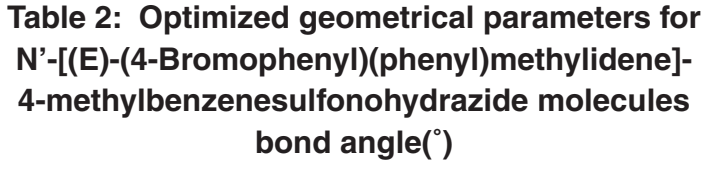

\begin{tabular}{|c|c|c|c|}
\hline $\begin{array}{l}\text { S. } \\
\text { No. }\end{array}$ & Bond angle & Experimental & $\begin{array}{l}\text { B3LYP } \\
\text { STO-3gG }\end{array}$ \\
\hline 1 & O1-S1-O2 & 119.55 & 122.0077 \\
\hline 2 & O1-S1-N2 & 107.91 & 105.7495 \\
\hline 3 & O1-S1-C14 & 107.62 & 111.4247 \\
\hline 4 & O2-S1-N2 & 104.08 & 111.3415 \\
\hline 5 & O2-S1-C14 & 111.01 & 107.7479 \\
\hline 6 & N2-S1-C14 & 105.77 & 95.3221 \\
\hline 7 & N2-N1-C7 & 117.06 & 116.4365 \\
\hline 8 & S1-N2-N1 & 111.23 & 104.0353 \\
\hline 9 & $\mathrm{~S} 1-\mathrm{N} 2-\mathrm{H} 1 \mathrm{~N} 2$ & 111.79 & 102.2319 \\
\hline 10 & N1-N2-H1N2 & 117.59 & 107.2695 \\
\hline 11 & $\mathrm{H} 1 \mathrm{~A}-\mathrm{C} 1-\mathrm{C} 2$ & 119.50 & 119.3254 \\
\hline 12 & $\mathrm{H} 1 \mathrm{~A}-\mathrm{C} 1-\mathrm{C} 6$ & 119.52 & 119.6576 \\
\hline 13 & C2-C1-C6 & 120.98 & 121.0148 \\
\hline 14 & $\mathrm{C} 1-\mathrm{C} 2-\mathrm{H} 2 \mathrm{~A}$ & 120.72 & 119.9058 \\
\hline 15 & C1-C2-C3 & 118.52 & 120.2135 \\
\hline 16 & $\mathrm{H} 2 \mathrm{~A}-\mathrm{C} 2-\mathrm{C} 3$ & 120.75 & 119.88 \\
\hline 17 & Br1-C3-C2 & 118.19 & 120.3839 \\
\hline 18 & Br1-C3-C4 & 119.81 & 120.2469 \\
\hline 19 & C2-C3-C4 & 122.00 & 119.3691 \\
\hline 20 & C3-C4-H4A & 120.60 & 119.8394 \\
\hline 21 & C3-C4-C5 & 118.81 & 120.0662 \\
\hline 22 & $\mathrm{H} 4 \mathrm{~A}-\mathrm{C} 4-\mathrm{C} 5$ & 120.59 & 120.0943 \\
\hline 23 & C4-C5-H5A & 119.66 & 120.5689 \\
\hline 24 & C4-C5-C6 & 120.76 & 121.1995 \\
\hline 25 & H5A-C5-C6 & 119.58 & 118.231 \\
\hline 26 & C1-C6-C5 & 118.90 & 118.1346 \\
\hline 27 & C1-C6-C7 & 120.72 & 122.0165 \\
\hline 28 & C5-C6-C7 & 120.38 & 119.8369 \\
\hline 29 & N1-C7-C6 & 115.65 & 115.4307 \\
\hline 30 & N1-C7-C8 & 124.75 & 125.1912 \\
\hline 31 & C6-C7-C8 & 119.60 & 119.3776 \\
\hline 32 & C7-C8-C9 & 119.94 & 121.1273 \\
\hline 33 & C7-C8-C13 & 120.67 & 120.3213 \\
\hline 34 & C9-C8-C13 & 119.36 & 118.5471 \\
\hline 35 & C8-C9-H9A & 119.87 & 119.3914 \\
\hline 36 & C8-C9-C10 & 120.20 & 120.6594 \\
\hline 37 & H9A-C9-C10 & 119.94 & 119.9437 \\
\hline 38 & C9-C10-H10A & 120.05 & 119.7477 \\
\hline 39 & C9-C10-C11 & 119.86 & 120.1663 \\
\hline 40 & H10A-C10-C11 & 120.08 & 120.086 \\
\hline 41 & C10-C11-H11A & 119.77 & 120.1319 \\
\hline 42 & C10-C11-C12 & 120.42 & 119.7757 \\
\hline
\end{tabular}

\begin{tabular}{llll}
43 & H11A-C11-C12 & 119.81 & 120.0925 \\
44 & C11-C12-H12A & 120.07 & 120.0209 \\
45 & C11-C12-C13 & 119.80 & 120.1798 \\
46 & H12A-C12-C13 & 120.13 & 119.7992 \\
47 & C8-C13-C12 & 120.32 & 120.6705 \\
48 & C8-C13-H13A & 119.82 & 119.3903 \\
49 & C12-C13-H13A & 119.86 & 119.9368 \\
50 & S1-C14-C15 & 120.78 & 116.8293 \\
51 & S1-C14-C19 & 117.89 & 120.9216 \\
52 & C15-C14-C19 & 120.99 & 122.2344 \\
53 & C14-C15-H15A & 120.72 & 119.2184 \\
54 & C14-C15-C16 & 118.49 & 118.8048 \\
55 & H15A-C15-C16 & 120.78 & 121.9758 \\
56 & C15-C16-H16A & 119.26 & 119.7779 \\
57 & C15-C16-C17 & 121.50 & 120.6102 \\
58 & H16A-C16-C17 & 119.24 & 119.6115 \\
59 & C16-C17-C18 & 118.49 & 119.0239 \\
60 & C16-C17-C20 & 121.27 & 120.5254 \\
61 & C18-C17-C20 & 120.22 & 120.444 \\
62 & C17-C18-H18A & 119.59 & 119.5142 \\
63 & C17-C18-C19 & 120.69 & 120.9404 \\
64 & H18A-C18-C19 & 119.73 & 119.5452 \\
65 & C14-C19-C18 & 119.74 & 118.3842 \\
66 & C14-C19-H19A & 120.16 & 120.7595 \\
67 & C18-C19-H19A & 120.10 & 120.8558 \\
68 & C17-C20-H20A & 109.50 & 110.7846 \\
69 & C17-C20-H20B & 109.52 & 110.3626 \\
70 & C17-C20-H20C & 109.42 & 110.8128 \\
71 & H20A-C20- & 109.44 & 107.9849 \\
72 & H20B & & \\
73 & H20A-C20- & 109.42 & 108.8753 \\
& H20C & & \\
\hline & & & 107.9249 \\
\hline
\end{tabular}

charge transfers in the chemical reaction [10, 11]. We have examined the Mulliken atomic charges in solution (Methanol) in Table 5. The Mulliken atomic charges calculated at the B3LYP STO-3gG. It is worthy to mention that $\mathrm{C} 6, \mathrm{C} 7$, and $\mathrm{C} 17$ atoms of N'[(E)-(4-Bromophenyl)(phenyl)methylidene]-4-methyl benzene sulfonohydrazide molecules exhibit positive charge, while C1, C2, C3, C4, C5, C8, C9, C10, C11, C12, C13, C14, C15, C16, C18, C19, and C20 atoms exhibits negative charges, Oxygen $\mathrm{O} 1$ and $\mathrm{O} 2$ has a maximum negative charges -0.46224 and -0.47788 for this values B3LYP STO-3gG basis set. The maximum positive atomic charges (1.235288) are obtained for $\mathrm{S} 1$ which is sulfonate present in the functional group $\mathrm{SO}_{3}$. The positive atomic charges 


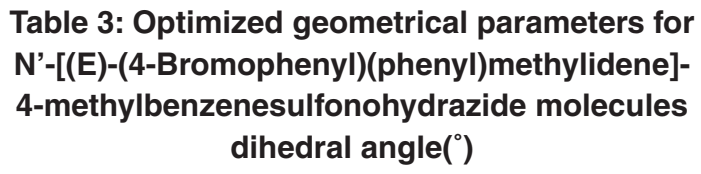
dihedral angle $\left({ }^{\circ}\right)$

\begin{tabular}{|c|c|c|c|}
\hline $\begin{array}{l}\text { S. } \\
\text { No. }\end{array}$ & $\begin{array}{l}\text { Dihedral } \\
\text { angle }\end{array}$ & Experimental & $\begin{array}{l}\text { B3LYP } \\
\text { STO-3gG }\end{array}$ \\
\hline 1 & O1-S1-N2-N1 & -70.31 & 175.4735 \\
\hline 2 & O1-S1-N2-H1N2 & 156.02 & 63.924 \\
\hline 3 & O2-S1-N2-N1 & 161.70 & 40.9306 \\
\hline 4 & O2-S1-N2-H1N2 & 28.03 & -70.6188 \\
\hline 5 & C14-S1- N2-N1 & 44.64 & -70.4822 \\
\hline 6 & C14-S1- N2-H1N2 & -89.03 & 177.9683 \\
\hline 7 & O1-S1-C14-C15 & -167.92 & -142.9805 \\
\hline 8 & O1-S1-C14-C19 & 18.69 & 35.6574 \\
\hline 9 & O2-S1-C14-C15 & -35.36 & -6.6428 \\
\hline 10 & O2-S1-C14-C19 & 151.25 & 171.9951 \\
\hline 11 & N2-S1-C14-C15 & 76.93 & 107.7873 \\
\hline 12 & N2-S1-C14-C19 & -96.46 & -73.5748 \\
\hline 13 & C7-N1-N2-S1 & -164.95 & -137.9619 \\
\hline 14 & C7-N1-N2-H1N2 & -34.23 & -30.1186 \\
\hline 15 & N2-N1-C7-C6 & 173.73 & -177.3678 \\
\hline 16 & N2-N1-C7-C8 & -6.85 & 2.3895 \\
\hline 17 & $\mathrm{H} 1 \mathrm{~A}-\mathrm{C} 1-\mathrm{C} 2-\mathrm{H} 2 \mathrm{~A}$ & 0.38 & 0.4516 \\
\hline 18 & $\mathrm{H} 1 \mathrm{~A}-\mathrm{C} 1-\mathrm{C} 2-\mathrm{C} 3$ & -179.68 & -179.2415 \\
\hline 19 & $\mathrm{C} 6-\mathrm{C} 1-\mathrm{C} 2-\mathrm{H} 2 \mathrm{~A}$ & -179.62 & 179.912 \\
\hline 20 & C6-C1-C2-C3 & 0.31 & 0.2188 \\
\hline 21 & $\mathrm{H} 1 \mathrm{~A}-\mathrm{C} 1-\mathrm{C} 6-\mathrm{C} 5$ & -178.81 & 178.9231 \\
\hline 22 & $\mathrm{H} 1 \mathrm{~A}-\mathrm{C} 1-\mathrm{C} 6-\mathrm{C} 7$ & 1.98 & 0.1842 \\
\hline 23 & C2-C1-C6-C5 & 1.20 & -0.5355 \\
\hline 24 & C2-C1-C6-C7 & -178.01 & -179.2744 \\
\hline 25 & $\mathrm{C} 1-\mathrm{C} 2-\mathrm{C} 3-\mathrm{Br} 1$ & 179.67 & -179.9516 \\
\hline 26 & C1-C2-C3-C4 & -1.09 & 0.1947 \\
\hline 27 & $\mathrm{H} 2 \mathrm{~A}-\mathrm{C} 2-\mathrm{C} 3-\mathrm{Br} 1$ & -0.40 & 0.3552 \\
\hline 28 & H2A-C2-C3-C4 & 178.85 & -179.4986 \\
\hline 29 & $\mathrm{Br} 1-\mathrm{C} 3-\mathrm{C} 4-\mathrm{H} 4 \mathrm{~A}$ & -0.47 & 0.0194 \\
\hline 30 & Br1-C3-C4-C5 & 179.54 & 179.8679 \\
\hline 31 & C2-C3-C4-H4A & -179.70 & 179.8733 \\
\hline 32 & C2-C3-C4-C5 & 0.31 & -0.2781 \\
\hline 33 & C3-C4-C5-H5A & -178.75 & 179.6607 \\
\hline 34 & C3-C4-C5-C6 & 1.26 & -0.0499 \\
\hline 35 & H4-C4-C5-H5A & 1.26 & -0.4911 \\
\hline 36 & H4A-C4-C5-C6 & -178.73 & 179.7983 \\
\hline 37 & C4-C5-C6-C1 & -2.00 & 0.4515 \\
\hline 38 & $\mathrm{C} 4-\mathrm{C} 5-\mathrm{C} 6-\mathrm{C} 7$ & 177.21 & 179.2188 \\
\hline 39 & H5A-C5-C6-C1 & 178.01 & -179.2656 \\
\hline 40 & H5A-C5-C6-C7 & -2.78 & -0.4983 \\
\hline 41 & C1-C6-C7-N1 & 157.08 & 164.5239 \\
\hline 42 & $\mathrm{C} 1-\mathrm{C} 6-\mathrm{C} 7-\mathrm{C} 8$ & -22.38 & -15.2485 \\
\hline
\end{tabular}

\begin{tabular}{|c|c|c|c|}
\hline 43 & C5-C6-C7-N1 & -22.12 & -14.194 \\
\hline 44 & $\mathrm{C} 5-\mathrm{C} 6-\mathrm{C} 7-\mathrm{C} 8$ & 158.43 & 166.0336 \\
\hline 45 & $\mathrm{~N} 1-\mathrm{C} 7-\mathrm{C} 8-\mathrm{C} 9$ & -58.22 & -51.7809 \\
\hline 46 & N1-C7-C8-C13 & 123.45 & 127.4563 \\
\hline 47 & $\mathrm{C} 6-\mathrm{C} 7-\mathrm{C} 8-\mathrm{C} 9$ & 121.18 & 127.9675 \\
\hline 48 & C6-C7-C8-C13 & -57.15 & -52.7952 \\
\hline 49 & C7-C8-C9-H9A & 1.75 & -1.3717 \\
\hline 50 & C7-C8-C9-C10 & -178.27 & 179.4752 \\
\hline 51 & C13-C8- C9-H9A & -179.90 & 179.3778 \\
\hline 52 & C13-C8- C9-C10 & 0.09 & 0.2248 \\
\hline 53 & C7-C8-C13-C12 & 179.65- & -179.1699 \\
\hline 54 & C7-C8-C13-H13A & -0.39 & 0.2613 \\
\hline 5 & C9-C8-C13-C12 & 1.31 & 0.0868 \\
\hline 56 & C9-C8-C13-H13A & -178.74 & 179.518 \\
\hline 57 & C8-C9-C10-H10A & 178.71 & 179.6285 \\
\hline 58 & C8-C9-C10-C11 & -1.30 & -0.37 \\
\hline 59 & $\mathrm{H} 9 \mathrm{~A}-\mathrm{C} 9-\mathrm{C} 10-\mathrm{H} 10 \mathrm{~A}$ & -1.31 & 0.4801 \\
\hline 50 & H9A-C9-C10-C11 & 178.69 & -179.5184 \\
\hline 61 & C9-C10- C11-H11A & -178.91 & -179.7863 \\
\hline 62 & C9-C10- C11-C12 & 1.11 & 0.2003 \\
\hline 53 & $\begin{array}{l}\text { H10A-C10- } \\
\text { C11-H11A }\end{array}$ & 1.08 & 0.2151 \\
\hline 4 & $\begin{array}{l}\text { H10A-C10- } \\
\text { C11-C12 }\end{array}$ & -178.89 & -179.7982 \\
\hline 65 & $\begin{array}{l}\text { C10-C11- } \\
\text { C12-H12A }\end{array}$ & -179.75 & -179.788 \\
\hline 66 & C10-C11-C12-C13 & 0.28 & 0.1101 \\
\hline 7 & $\begin{array}{l}\text { H11A-C11- } \\
\text { C12-H12A }\end{array}$ & 0.27 & 0.1986 \\
\hline 68 & $\begin{array}{l}\text { H11A-C11- } \\
\text { C12-C13 }\end{array}$ & -179.70 & -179.9032 \\
\hline 69 & C11-C12-C13-C8 & -1.50 & -0.2544 \\
\hline 70 & $\begin{array}{l}\text { C11-C12- } \\
\text { C13-H13A }\end{array}$ & 178.55 & -179.6824 \\
\hline 71 & $\begin{array}{l}\text { H12A-C12- } \\
\text { C13-C8 }\end{array}$ & 178.53 & 179.644 \\
\hline 72 & $\begin{array}{l}\text { H12A-C12- } \\
\text { C13-H13A }\end{array}$ & -1.42 & 0.2159 \\
\hline 73 & S1-C14-C15-H15A & 8.72 & -0.528 \\
\hline 74 & S1-C14-C15-C16 & -171.29 & 179.1197 \\
\hline 75 & $\begin{array}{l}\text { C19-C14- } \\
\text { C15-H15A }\end{array}$ & -178.10 & -179.1466 \\
\hline 76 & C19-C14-C15-C16 & 1.89 & 0.5011 \\
\hline 77 & S1-C14-C19-C18 & 171.17 & -179.033 \\
\hline 78 & S1-C14- C19-H19A & -8.83 & 0.7163 \\
\hline 79 & C15-C14-C19-C18 & -2.21 & -0.47 \\
\hline 80 & $\begin{array}{l}\text { C15-C14- } \\
\text { C19-H19A }\end{array}$ & 177.79 & 179.2793 \\
\hline 8 & $\begin{array}{l}\text { C14-C15- } \\
\text { C16-H16A }\end{array}$ & -179.22 & -179.9372 \\
\hline
\end{tabular}




\begin{tabular}{|c|c|c|c|}
\hline 82 & C14-C15- & 0.80 & -0.1649 \\
\hline & C16-C17 & & \\
\hline 83 & H15A-C15- & 0.77 & -0.2997 \\
\hline & $\mathrm{C} 16-\mathrm{H} 16 \mathrm{~A}$ & & \\
\hline 84 & H15A-C15- & -179.21 & 179.4726 \\
\hline & C16-C17 & & \\
\hline 85 & C15-C16-C17-C18 & -3.10 & -0.1814 \\
\hline 86 & C15-C16-C17-C20 & 175.50 & 178.8862 \\
\hline 87 & H16A-C16- & 176.92 & 179.5913 \\
\hline & C17-C18 & & \\
\hline 88 & H16A-C16- & -4.48 & -1.3411 \\
\hline & $\mathrm{C} 17-\mathrm{C} 20$ & & \\
\hline 89 & C16-C17- & -177.25 & -179.6164 \\
\hline & $\mathrm{C} 18-\mathrm{H} 18 \mathrm{~A}$ & & \\
\hline 90 & C16-C17-C18-C19 & 2.78 & 0.2121 \\
\hline 91 & C20-C17- & 4.14 & 1.3152 \\
\hline & $\mathrm{C} 18-\mathrm{H} 18 \mathrm{~A}$ & & \\
\hline 92 & C20-C17-C18-C19 & -175.84 & -178.8563 \\
\hline 93 & C16-C17- & 18.20 & 27.7399 \\
\hline & $\mathrm{C} 20-\mathrm{H} 20 \mathrm{~A}$ & & \\
\hline 94 & C16-C17- & -101.81 & -91.8077 \\
\hline & $\mathrm{C} 20-\mathrm{H} 2 \mathrm{OB}$ & & \\
\hline 95 & C16-C17- & 138.13 & 148.7013 \\
\hline & $\mathrm{C} 20-\mathrm{H} 20 \mathrm{C}$ & & \\
\hline 96 & C18-C17- & -163.22 & -153.2058 \\
\hline & C2O-H2OA & & \\
\hline 97 & C18-C17- & 76.77 & 87.2466 \\
\hline & $\mathrm{C} 20-\mathrm{H} 20 \mathrm{~B}$ & & \\
\hline 98 & C18-C17- & -43.29 & -32.2444 \\
\hline & $\mathrm{C} 20-\mathrm{H} 20 \mathrm{C}$ & & \\
\hline 99 & C17-C18-C19-C14 & -0.19 & 0.1045 \\
\hline 100 & C17-C18- & 179.81 & -179.6445 \\
\hline & $\mathrm{C} 19-\mathrm{H} 19 \mathrm{~A}$ & & \\
\hline 101 & H18A-C18- & 179.84 & 179.9329 \\
\hline & C19-C14 & & \\
\hline 102 & H18A-C18- & -0.16 & 0.1839 \\
\hline & C19-H19A & & \\
\hline
\end{tabular}

are observed (0.027547) for bromine atoms. The magnitude of hydrogen atomic charges is hydrogen atomic charges are found to be only positive and negative charges obtain, this listed given in the Table 5 for this B3LYP STO-3gG basis sets for the N'-[(E)-(4-Bromophenyl)(phenyl)methylidene]-4methyl benzene sulfonohydrazide molecules. The atomic charges plotted B3LYP STO-3gG basis set has been shown in Fig. 7. The nitrogen atoms presence of negative charges are $\mathrm{N} 1(-0.15703)$ and N2 (-0.45769) atoms. The above result shows that the natural atomic charges are more sensitive to the
Table 4: Comparison of HOMO, LUMO, energy gaps (e HOMO -LUMO), and related molecular properties of N'-[(E)-(4-Bromophenyl)(phenyl) methylidene]-4-methyl benzene sulfonohydrazide molecules (a.u.)

\begin{tabular}{ll}
\hline Molecular properties & B3LYP STO-3gG \\
\hline EHOMO & -0.11757 \\
ELUMO & 0.01354 \\
$\Delta$ EHOMO-LUMO gap (a.u.) & -0.10403 \\
Ionisation Potential $(\mathrm{I})$ & \\
Electron affinity $(\mathrm{A})$ & 0.11757 \\
& -0.01354 \\
Global Hardness $(\eta)$ & -0.0646 \\
Chemical potential $(\mu)$ & -0.0520 \\
Global Electrophilicity $(\omega)$ & 0.02089 \\
\hline
\end{tabular}

charges in the molecular structure the Mullikan's net charges.

\section{HOMO-LUMO analysis}

A deeper understand of chemical reactivity can be gained by this electronic absorption corresponds to the transition from the ground state to the first excited state and it is mainly described by one electron excitation from the highest occupied molecular orbital (HOMO) to the lowest unoccupied molecular orbital (LUMO) ${ }^{12,13}$. HOMO represents the ability to donate an electron and LUMO represent the ability to obtain an electron. The HOMO is delocalized over the Bromine substituted two benzene ring and bridge over the N-NH group. The LUMO is located on the Bromo benzophenone and tosylhydrazide group. Consequently, the HOMOLUMO transition implies an electron density transfer from, the more aromatic part of the $\pi$-conjucated system including the electron donor group to its more quinonid side and mainly to the electron with drawing end the frontier molecular orbital's of 
Table 5: The charge distribution calculated by the Mulliken method for N'-[(E)-(4Bromophenyl)(phenyl)methylidene]-4methylbenzenesulfonohydrazide molecules

\begin{tabular}{|c|c|}
\hline Atoms & Charges \\
\hline $\mathrm{Br} 1$ & 0.027547 \\
\hline S1 & 1.235288 \\
\hline 01 & -0.46224 \\
\hline $\mathrm{O} 2$ & -0.47788 \\
\hline N1 & -0.15703 \\
\hline N2 & -0.45769 \\
\hline H1N2 & 0.271619 \\
\hline C1 & -0.10475 \\
\hline $\mathrm{H} 1 \mathrm{~A}$ & 0.110807 \\
\hline $\mathrm{C} 2$ & -0.11313 \\
\hline $\mathrm{H} 2 \mathrm{~A}$ & 0.112495 \\
\hline C3 & -0.04562 \\
\hline $\mathrm{C} 4$ & -0.11412 \\
\hline $\mathrm{H} 4 \mathrm{~A}$ & 0.111453 \\
\hline C5 & -0.09862 \\
\hline $\mathrm{H} 5 \mathrm{~A}$ & 0.117707 \\
\hline C6 & 0.010596 \\
\hline $\mathrm{C7}$ & 0.070907 \\
\hline $\mathrm{C8}$ & -0.00651 \\
\hline $\mathrm{C9}$ & -0.10659 \\
\hline $\mathrm{H} 9 \mathrm{~A}$ & 0.114875 \\
\hline C10 & -0.10129 \\
\hline $\mathrm{H} 10 \mathrm{~A}$ & 0.109164 \\
\hline C11 & -0.10274 \\
\hline $\mathrm{H} 11 \mathrm{~A}$ & 0.107332 \\
\hline C12 & -0.10209 \\
\hline $\mathrm{H} 12 \mathrm{~A}$ & 0.107313 \\
\hline C13 & -0.11076 \\
\hline $\mathrm{H} 13 \mathrm{~A}$ & 0.107218 \\
\hline C14 & -0.13433 \\
\hline C15 & -0.11229 \\
\hline $\mathrm{H} 15 \mathrm{~A}$ & 0.10977 \\
\hline C16 & -0.10761 \\
\hline $\mathrm{H} 16 \mathrm{~A}$ & 0.106391 \\
\hline C17 & 0.039951 \\
\hline C18 & -0.10834 \\
\hline $\mathrm{H} 18 \mathrm{~A}$ & 0.10838 \\
\hline C19 & -0.10236 \\
\hline $\mathrm{H} 19 \mathrm{~A}$ & 0.123402 \\
\hline C20 & -0.34109 \\
\hline $\mathrm{H} 2 \mathrm{OA}$ & 0.11906 \\
\hline H2OB & 0.123953 \\
\hline H2OC & 0.121847 \\
\hline
\end{tabular}

N'-[(E)-(4-Bromophenyl) (phenyl)methylidene]-4methyl benzene sulfonohydrazide is shown in Fig. 8. The energy value of $\mathrm{HOMO}$ is computed -0.11757 a.u. and LUMO is 0.01354 a.u. The energy gap is -0.10403 a.u. in for N'-[(E)-(4-Bromophenyl)(phenyl) methylidene]-4-methyl benzene sulfonohydrazide molecules, respectively. Surface for the Frontier orbitals are drawn to understand the bonding scheme of N'-[(E)-(4-Bromophenyl)(phenyl)methylidene]-4methyl benzene sulfonohydrazide molecules. We examine the two important molecular orbital's (MO) for N'-[(E)-(4-Bromophenyl)(phenyl)methylidene]-4methyl benzene sulfonohydrazide molecules: highest occupied MOs and lowest unoccupied and MOs which we denote HOMO and LUMO respectively.

The calculated Self Consistent Field (SCF) energy of N'-[(E)-(4-Bromophenyl)(phenyl) methylidene]-4-methylbenzenesulfonohydrazide is -3958.29016076 a.u. at B3LYP STO-3gG. The $\mathrm{HOMO}$ and LUMO energy gap explains the fact that eventual charge transfer interaction is taking place within the molecules.

\section{HOMO-LUMO energy gap and related molecular properties}

The HOMO, LUMO and HOMO-LUMO energy gap of N'-[(E)-(4-Bromophenyl)(phenyl) methylidene]-4-methyl benzene sulfonohydrazide molecules in the B3LYP STO-3gG basis set has been calculated. The HOMO-LUMO energy gap reveals that the energy gap reflects the chemical activity of the molecule. Associated within the framework of SCF MO theory the ionization energy and electron affinity can be expressed through HOMO and LUMO orbital energies as $\mathrm{I}=-\mathrm{E}_{\text {Hомо }}$ and $A=-E_{\text {LUMO }}$. The hardness corresponds to the gap between the HOMO and LUMO orbital energies. The larger the HOMO-LUMO energy gaps the harder the molecules ${ }^{14}$. The global hardness, $\eta=1 / 2\left(E_{\text {LUMO }}\right.$ - $\left.\mathrm{E}_{\text {номо }}\right)$. The hardness has been associated with the stability of chemical system. The electron affinity can be used in combination with ionization energy to give electronic chemical potential, $\mu=1 / 2\left(E_{\text {номо }}+\right.$ $\left.E_{\text {LUMO }}\right)$. The global electrophilicity index, $\omega=\mu^{2} / 2 \eta$ is also calculated and listed in Table 4.

\section{Molecular electrostatic potential (MEP)}

The 3D plots of molecular electrostatic potential (MEP) of N'-[(E)-(4-Bromophenyl)(phenyl) 


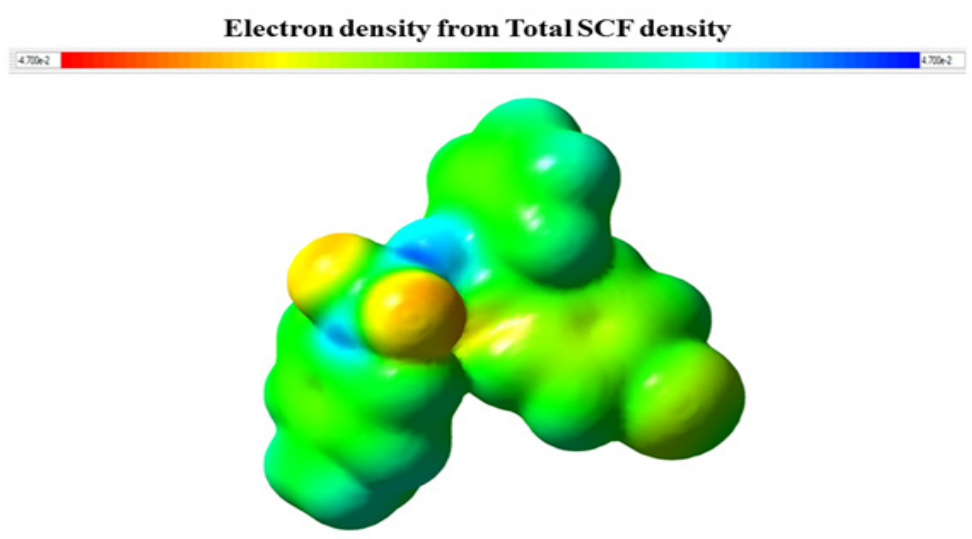

Fig. 10: The total electron density surface mapped with electrostatic potentialN'-[(E)-(4Bromophenyl)(phenyl)methylidene]-4-methylbenzenesulfonohydrazide molecules

methylidene]-4-methyl benzene sulfonohydrazide molecule is illustrated in Fig. 9. The MEP is a plot of electrostatic potential mapped onto the constant electron density surface. The MEP surface superimposed on top of the total energy density. The MEP is a useful property to study reactivity given that an approaching electrophile will be attracted to negative region (where the electron distribution effect is dominant). In the majority of the MEPs, while the maximum negative region which preferred site of for electrophilic attack indication as red colour, the maximum positive region which preferred site for nucleophilic attack symptoms as blue colour. The importance of MEP lies in the fact that it simultaneous displays molecular size, shape as well as positive, negative and neutral electrostatic potential regions in terms of colour grading (Fig. 9) and is very useful in research of molecular structure with its physiochemical property relationship ${ }^{15,16}$. The resulting surface simultaneously displays molecular size and shape and electrostatic potential value.

The different values of the electrostatic potential at the surface are represented by different colours. The potential increases in the order red $<$ orange < yellow < green $<$ blue. The colour code of these maps is the range between the HOMO - 1.437 a.u. (Deepest red) to 1.437 a.u. (Deepest blue) and LUMO is - 1.521 a.u. (Deepest red) to 1.521 a.u in N'-[(E)-(4-Bromophenyl)(phenyl) methylidene]-4-methyl benzene sulfonohydrazide molecules. Whereas blue colour indicates the strongest attraction and red colour indicates the strongest repulsion. The regions of negative $V(r)$ are usually associated with the lone pair of electro native atoms. The contour map of electrostatic potential of the N'-[(E)-(4-Bromophenyl)(phenyl)methylidene]4-methyl benzene sulfonohydrazide molecule has been constructed by the B3LYP STO-3gG basis set is shown in Fig. 10also confirms the different negative (-4.700 a.u.) and positive (-4.700 a.u.) potential sites of the molecules in accordance with the total electron density surface.

\section{CONCLUSION}

In present investigation, N'-[(E)-(4Bromophenyl)(phenyl)methylidene]-4-methyl benzene sulfonohydrazide crystals were grown by slow evaporation method. The X-ray single crystal structural refinement indicated monoclinic structure and good crystalline quality. The of $\mathrm{HOMO}$ LUMO analyses, energy value of $\mathrm{HOMO}$ was computed -0.11757 a.u. and LUMO was 0.01354 a.u. and HOMO-LUMO energy was -0.10403 a.u. The molecular electrostatic potential result reflected, the surface simultaneously displays molecular size and shape and electrostatic potential value. The total electron density surface mapped with electrostatic potential have different negative (-4.700 a.u.) and positive (-4.700 a.u.) potential sites. The Mulliken atomic charges calculated at the B3LYP STO-3gG. It was worthy to mention that $\mathrm{C} 6, \mathrm{C} 7$, and $\mathrm{C} 17$ atoms of N'-[(E)-(4-Bromophenyl)(phenyl)methylidene]-4methyl benzene sulfonohydrazide molecules exhibit positive charge, while C1, C2, C3, C4, C5, C8, C9, C10, C11, C12, C13, C14, C15, C16, C18, C19, and $\mathrm{C} 20$ atoms exhibited negative charges, Oxygen $\mathrm{O} 1$ 
and $\mathrm{O} 2$ have been a maximum negative charges -0.46224 and -0.47788 for those values B3LYP STO$3 \mathrm{gG}$ basis set. The theoretical molecular structures of N'-[(E)-(4-Bromophenyl)(phenyl)methylidene]-4methyl benzene sulfonohydrazide were determined by the B3LYP STO-3gG. It is suggested this crystal will be used for nonlinear electro optic field.

\section{ACKNOWLEDGEMENT}

One of the authors P. M acknowledges the support rendered by UGC-BSR, under Start-up grant.

\section{REFERENCES}

1 Carroll, G.T.; Turro, N.J.; Koberstein, J.T.; J. Colloid Interf. Science 2010, 351, 556-560. Ajani, O.O.; Obafemi, C.A.; Nwinyi, O.C.; Akinpelu, D.A.; Bioorg. Med. Chem., 2010,18, 214-221.

3 Gerdemann, C.; Eicken, C.; Krebs, B.; Acc. Chem. Res., 2002,35, 183-191.

$4 \quad$ Kutzke, H.; Klapper, H.; Hammond, R.B.; Roberts, K.J.; ActaCryst. B, 2010,56, 486496.

5 Zhang, W.G.; ActaCryst.E 2011,67, 02331-6.

6 Shen, X.H.; Zhu, L.X.; Shao, L.J.; Zhu, Z.F.; ActaCryst. E 2013, 68, 0297-1-5.

7 Khanum, S.A.; Venu, T.D.; Shashikanth, S.; Firdouse, A.; Bioorg. Med. Chem. Lett., 2004,12, 2093-2095.

8 Balaji, J.; Prabu, S.; Xavier, J.J.F.; Srinivasan, P.; ActaCryst. E 2015, 71, 045-046.

9 Gaussian 09, Revision E.01, Frisch, M.J.; et al., Gaussian, Inc., Wallingford CT, 2009.

10 K. Jug, Z.B. Maksic, in; Z.B. Maksic (Ed.), Theortical Model of chemical Bonding, Part 3, Springer, Berlin, 1991.

11 Flishzer, S.; Charge Distributions and chemical Effects, Spinger, New York, 1983.

12 Kavitha, E.; Sundaraganesan, N.; Sebatain, S.; Indian. J. Pure Applied Phys., 2010,48, 20-30.

13 Prasad, O.; Sinha, L.; Kumar, N.; J. Atom mol. Sci. 2010,1,201-214.

14 Rastogi, K.; Palafox, M.A.; Tanwar, R.P.; Mittal, L.; SpectrochimActa A., 2002,58, 19872004.

15 Murry, J.S.; Sen, K.; Molecular Electrostatic Potential, Concepts and Application, Elsevier, Amsterdam, 1996.

16 Scrocco, E.; Tomasi J.; in: P. Lowdin (Ed.), Advances in Quantum Chemistry, Academic press, New York, 1978. 